\title{
Clinical significance of circulating tumor cells and metabolic signatures in lung cancer after surgical removal
}

\author{
Dawei Yang ${ }^{1}$, Xiaofang Yang ${ }^{2}$, Yang $\mathrm{Li}^{1}$, Peige Zhao ${ }^{3}$, Rao Fu${ }^{1}$, Tianying Ren ${ }^{1}$, Ping Hu${ }^{1}$, Yaping $\mathrm{Wu}^{1}$, \\ Hongjun Yang ${ }^{4^{*}}$ and $\mathrm{Na} \mathrm{Guo}{ }^{2,5^{*}}$ (1)
}

\begin{abstract}
Background: Lung cancer (LC) remains the deadliest form of cancer globally. While surgery remains the optimal treatment strategy for individuals with early-stage LC, what the metabolic consequences are of such surgical intervention remains uncertain.

Methods: Negative enrichment-fluorescence in situ hybridization (NE-FISH) was used in an effort to detect circulating tumor cells (CTCs) in pre- and post-surgery peripheral blood samples from 51 LC patients. In addition, targeted metabolomics analyses, multivariate statistical analyses, and pathway analyses were used to explore surgery-associated metabolic changes.

Results: $L C$ patients had significantly higher CTC counts relative to healthy controls with $66.67 \%$ of LC patients having at least 1 detected CTC before surgery. CTC counts were associated with clinical outcomes following surgery. In a targeted metabolomics analysis, we detected 34 amino acids, 147 lipids, and 24 fatty acids. When comparing LC patients before and after surgery to control patients, metabolic shifts were detected via PLS-DA and pathway analysis. Further surgery-associated metabolic changes were identified when comparing $L A$ ( $L C$ patients after surgery) and $L B$ (LC patients before surgery) groups. We identified SM 42:4, Ser, Sar, Gln, and LPC 18:0 for inclusion in a biomarker panel for early-stage LC detection based upon an AUC of 0.965 (95\% Cl 0.900-1.000). This analysis revealed that SM 42:2, SM 35:1, PC (16:0/14:0), PC (14:0/16:1), Cer (d18:1/24:1), and SM 38:3 may offer diagnostic and prognostic benefits in LC.
\end{abstract}

Conclusions: These findings suggest that CTC detection and plasma metabolite profiling may be an effective means of diagnosing early-stage $\mathrm{LC}$ and identifying patients at risk for disease recurrence.

Keywords: Lung cancer, Circulating tumor cells (CTC), Metabolomics, Negative enrichment-fluorescence in situ hybridization (NE-FISH), Metabolic pathway analysis

*Correspondence: hongjun0420@vip.sina.com; quona5246@126.com

2 Experimental Research Center, China Academy of Chinese Medical Sciences, Beijing 100700, People's Republic of China

${ }^{4}$ Institute of Chinese Materia Medica, China Academy of Chinese Medical Sciences, Beijing 100700, People's Republic of China

Full list of author information is available at the end of the article

\section{Introduction}

Lung cancer (LC) is among the most common forms of cancer globally, with 2.1 million new cases and 1.8 million LC-related deaths in 2018 alone [1]. LC has a 5-year survival rate of $<20 \%$. This poor prognosis is attributable in part to the fact that many patients are only diagnosed when the disease is at an advanced stage, with metastases often being present. Standard treatments for LC at present include radiotherapy, chemotherapy, surgical

(c) The Author(s) 2020. This article is licensed under a Creative Commons Attribution 4.0 International License, which permits use, sharing, adaptation, distribution and reproduction in any medium or format, as long as you give appropriate credit to the original author(s) and the source, provide a link to the Creative Commons licence, and indicate if changes were made. The images or other third party material in this article are included in the article's Creative Commons licence, unless indicated otherwise in a credit line to the material. If material is not included in the article's Creative Commons licence and your intended use is not permitted by statutory regulation or exceeds the permitted use, you will need to obtain permission directly from the copyright holder. To view a copy of this licence, visit http://creativeco mmons.org/licenses/by/4.0/. The Creative Commons Public Domain Dedication waiver (http://creativecommons.org/publicdomain/ zero/1.0/) applies to the data made available in this article, unless otherwise stated in a credit line to the data. 
resection, and targeted therapeutic interventions [2, 3]. Surgical treatment of patients with early-stage (stage I/II) is associated with good long-term outcomes $[4,5]$. Surgery is also important for treating individuals with more advanced (stage III/IV) LC [6]. Recent surgical innovations such as video-assisted thoracoscopic surgery have also decreased the invasivity of these intervention protocols, thus allowing more patients to be candidates for surgical treatment [7].

A number of different cancer-associated materials can be detected in circulating peripheral blood, including tumor-derived exosomes, circulating tumor DNA (ctDNA), and circulating tumor cells (CTCs) [8]. Specific cancer-derived proteins including carcinoembryonic antigen (CEA) and alpha-fetoprotein (AFP) are similarly detectable, as well as circulating metabolites. Measurement of these markers can offer insight into disease progression in both primary tumor and metastatic compartments, and can also be used as a means of screening for and diagnosing early-stage cancers.

CTCs are released from primary or metastatic tumors into circulation [9]. Although these cells are extremely rare in most cancer patients, recent improvements in CTC capture, enrichment, and detection technologies have significantly improved the clinical feasibility of analyzing these cells to monitor tumor progression and therapeutic responsiveness [9-11]. CTC levels have been shown to be independently predictive of patient outcomes in patients with both non-small cell lung cancer (NSCLC) $[12,13]$ and small cell lung cancer (SCLC) $[14,15]$. Cancers can further be monitored in real time via single-cell-based analyses of these CTCs, thus offering a more direct insight into the metastatic progression of a given patient's cancer [16-18]. CTC detection may thus allow for the identification of those LC patients with a higher risk of disease recurrence, potentially allowing clinicians to determine which patients are most likely to benefit from adjuvant treatment following surgery.

Surgery is likely to alter metabolite profiles in LC patients such that metabolomic studies may allow for a more in-depth understanding of these operation-associated shifts. Metabolomic studies allow for the simultaneous analysis of thousands of different endogenous metabolites in a given biological sample. Previous studies have employed metabolomic approaches in studies of cancers of the lung, breast, prostate, bladder, and other tissues [19-21]. These metabolomic approaches have allowed for the identification of cancer-associated biomarkers and/or biomarkers associated with therapeutic responsiveness, thus allowing for the monitoring of these biomarkers in order to better understand disease progression [22]. Previous studies have sought to identify metabolite biomarkers associated with LC patient outcomes [23]. How surgical tumor resection affects these metabolite profiles, however, remains uncertain.

Metabolite profiling can be sensitively conducted using an ultra-performance liquid chromatography/mass spectrometry (UPLC/MS) platform [24, 25]. In the present study, we utilized ultra-performance liquid chromatography with quadrupole time-of-flight mass spectrometry (UPLC-QTOF/MS) and ultra-performance liquid chromatography-tandem mass spectrometry (UPLCMS/MS) approaches together with multivariate statistical approaches in order to explore shifts in LC patient metabolomic serum profiles in response to surgical intervention. We additionally quantified CTC frequencies in these patients before and after surgery and explored the prognostic relevance of metabolic and CTC signatures in this patient population.

\section{Materials and methods Chemicals and materials}

MS-grade methanol, formic acid, acetic acid and acetonitrile were purchased from Fisher scientific. Ultra-pure water $(18.2 \mathrm{M} \Omega$ ) was prepared with a Milli-Q water purification system (Millipore, Bedford, MA, USA). Glycine (Gly), $\beta$-alanine ( $\beta$-Ala), sarcosine (Sar), L-alanine (Ala), L-serine (Ser), L-proline (Pro), L-valine (Val), L-threonine (Thr), L-cysteine (Cys), taurine (Tau), L-leucine (Leu), $\gamma$-aminobutyric acid (GABA), L-isoleucine (Ile), trans-4-hydroxy-L-proline ( $\mathrm{t}$-Hyp), L-asparagine (Asn), L-aspartic Acid (Asp), L-ornithine (Orn), L-lysine (Lys), L-glutamic acid (Glu), L-methionine (Met), 1-methylL-histidine, 3-methyl-L-histidine, L-2-aminoadipic acid, L-phenylalanine (Phe), L-arginine (Arg), L-citrulline (Cit), L-tyrosine (Tyr), L-tryptophan (Trp), L-carnosine, folic acid (FA), kynurenic acid, pipecolinic acid were purchased from Sigma-Aldrich company (St. Louis, MO, USA); L-histidine (His) and glutamine (Gln) were purchased from TCI (Develoment Co., Ltd, Shanghai, China); Phenylalanine-d5 (Internal standard, IS) was purchased from Toronto Research Chemicals (YTO, Canada). The categories of lysophosphatidylcholine (LPC), phosphatidylcholine (PC), lysophosphatidylethanolamine (LPE), phosphatidylethanolamine (PE), sphingomyelin (SM), ceramide (Cer), were purchased from Avanti Polar Lipids company (Alabaster, AL, USA); triacylglycerol (TAG), free fatty Acid were purchased from Larodan company (Stockholm, Sweden). ISs of PC (19:0/19:0), LPC (19:0/0:0), PE (12:0/13:0), SM(d18:1/12:0), Cer (d18:1/17:0) were purchased from Avanti Polar Lipids company (Alabaster, AL, USA), TAG (15:0/15:0/15:0), FFA (C19:0), d3-FFA(C16:0) were purchased from Larodan company (Stockholm, Sweden). All other chemicals and reagents used were of analytical grade. 


\section{Study population}

Patients that presented to the Liaocheng People's Hospital LC clinic with suspected LC from September 2016-January 2018 were included in the present study. A total of 51 paired pre- and post-operative serum samples were collected from patients that had provided written informed consent to participate (Table 1). All patients were newly diagnosed and untreated. All cancer patients were confirmed by histopathological diagnosis. We excluded patients who recently received radiotherapy, chemotherapy or immunotherapy, and had non-pulmonary primary tumor. Patients with benign diseases were diagnosed by imaging, serum tests, and histopathology. Criteria for healthy donors included non-smoking; non-alcoholic; no family history of cancer; no tumors or other disease history; normal heart, liver, lung and brain; and normal results on routine tests for blood, urine, feces, tumor markers, renal and liver function, chest X-ray and electrocardiography. Following tumor resection, histopathological confirmation of LC diagnosis was made, and tumor stage and clinicohistopathological characteristics were analyzed. As a control group, 51 age- and sex-matched healthy volunteers were recruited. The Ethics Committee of Liaocheng People's Hospital approved this study, which was conducted in a manner consistent with the Declaration of Helsinki.

\section{Sample preparation}

Preoperative serum samples were collected from LC patients on the morning following hospitalization, while postoperative serum samples were collected on the last morning prior to discharge for each patient (7 days post-operation). For all sample collections, peripheral blood $(\sim 3.2 \mathrm{~mL})$ was collected in a Citratedextrose solution (ACD) Vacutainer tube (BD, NJ, USA), and samples were stored at room temperature (RT) prior to analysis for CTC levels. For metabolomics assays, an additional $2 \mathrm{~mL}$ of serum was collected for analysis, with control samples being collected contemporaneously.

Table 1 The relationship between patient demographics, clinical characteristics, and CTC levels

\begin{tabular}{|c|c|c|c|c|c|c|c|}
\hline \multirow[t]{2}{*}{ Characteristics } & \multirow[t]{2}{*}{$n$} & \multirow[t]{2}{*}{ Proportion (\%) } & \multicolumn{2}{|c|}{$\mathrm{CTC}<1$} & \multicolumn{2}{|c|}{$\mathrm{CTC} \geq 1$} & \multirow[t]{2}{*}{$P$} \\
\hline & & & $n$ & Proportion (\%) & $n$ & Proportion (\%) & \\
\hline \multicolumn{8}{|l|}{ Gender } \\
\hline Male & 29 & 56.86 & 7 & 24.14 & 22 & 75.86 & \multirow[t]{2}{*}{0.104} \\
\hline Female & 22 & 43.14 & 10 & 45.45 & 12 & 54.55 & \\
\hline \multicolumn{8}{|l|}{ Age } \\
\hline$\geq 60$ & 35 & 68.63 & 10 & 28.57 & 25 & 71.43 & \multirow[t]{2}{*}{0.602} \\
\hline$<60$ & 16 & 31.37 & 7 & 43.75 & 9 & 56.25 & \\
\hline \multicolumn{8}{|l|}{ Histology } \\
\hline Adenocarcinoma & 31 & 60.78 & 12 & 38.71 & 19 & 61.29 & \multirow[t]{3}{*}{0.904} \\
\hline Squamous & 16 & 31.37 & 4 & 25 & 12 & 75 & \\
\hline SCLC & 4 & 7.84 & 1 & 25 & 3 & 75 & \\
\hline \multicolumn{8}{|l|}{ Distant metastasis } \\
\hline $\mathrm{MO}$ & 49 & 96.08 & 16 & 32.65 & 33 & 67.35 & \multirow[t]{2}{*}{0.306} \\
\hline M1 & 2 & 3.92 & 1 & 50 & 1 & 50 & \\
\hline \multicolumn{8}{|l|}{ Tumor depth } \\
\hline $\mathrm{T} 1$ & 29 & 56.86 & 14 & 48.28 & 15 & 51.72 & \multirow[t]{4}{*}{0.141} \\
\hline $\mathrm{T} 2$ & 13 & 25.49 & 3 & 23.08 & 10 & 76.92 & \\
\hline T3 & 5 & 9.80 & 0 & 0 & 5 & 100 & \\
\hline $\mathrm{T} 4$ & 4 & 7.84 & 0 & 0 & 4 & 100 & \\
\hline \multicolumn{8}{|c|}{ Lymph node metastasis } \\
\hline Yes & 18 & 35.29 & 0 & 0 & 18 & 100 & \multirow[t]{2}{*}{0.003} \\
\hline No & 33 & 64.71 & 17 & 51.52 & 16 & 48.48 & \\
\hline \multicolumn{8}{|l|}{ TNM stage (UIUC) } \\
\hline । & 29 & 56.86 & 16 & 55.17 & 13 & 44.83 & \multirow[t]{4}{*}{0.001} \\
\hline$\|$ & 10 & 19.61 & 0 & 0 & 10 & 100 & \\
\hline III & 10 & 19.61 & 0 & 0 & 10 & 100 & \\
\hline IV & 2 & 3.92 & 1 & 50 & 1 & 50 & \\
\hline
\end{tabular}




\section{Clinical effect criteria}

RECIST (Response Evaluation Criteria in Solid Tumors) v1.1 was used for the evaluation of clinical responses. Responses were classified based upon tumor diameter and axillary lymph node status as follows: complete response $(\mathrm{CR})$, stable disease (SD), partial response $(\mathrm{PR})$, or progressive disease (PD). Clinical efficacy was considered to have been achieved when PR or CR was diagnosed. For analysis purposes, patients with $\mathrm{CR}$ or $\mathrm{PR}$ were incorporated into one analysis group $(\mathrm{CR}+\mathrm{PR})$ while patients with SD or PD were incorporated into a second group (SD + PD). Patients underwent follow-up through October 31, 2019, via phone call and outpatient visits. The time between surgery and the last diagnosis of recurrence was 21-37 months. A total of 14 LC patients suffered from disease recurrence, while 31 did not suffer from recurrence and 6 did not have sufficient available clinical information to determine whether disease had recurred.

\section{CTC detection}

Negative enrichment fluorescence in situ hybridization (NE-FISH) was used to detect CTCs as in our previous studies [26, 27]. Briefly, patient blood samples were washed and RBCs were lysed with the CS1 and CS2 buffers, respectively, followed by resuspension in a solution containing magnetic particles bound to anti-CD45 (Cyttel Biosciences INC., Jiangsu, China). Gradient centrifugation was then performed using the CS3 buffer (Cyttel) to separate samples, and a magnetic stand was used to collect the CTC-containing sample. This sample was then fixed and spread onto a slide, which was then dried for analysis. FISH was then performed using the chromosome centromere probe (CEP) $8+17$, with DAPI used to stain cell nuclei and AF594-conjugated anti-CD45 being used to stain cells. Slides were then imaged via microscopy (BX63, Olympus), and an IMSTAR high content screening device equipped with the PathfinderTM software (IMSTAR S.A., Paris, France) was used for image analysis. CTCs were cells that were found to be DAPI+/ CD45-/CEP+ (Fig. 1).

\section{UPLC-MS/MS}

Amino acids, lipids, and fatty acids were analyzed with a UPLC system (Waters ACQUITY) that contained a quaternary pump, an autosampler, a degasser, and a Xevo TQ-S micro mass spectrometer with an ESI ionization source (Waters, MA, USA). The amino acid analysis was conducted following sample separation with a Waters ACQUITY UPLC BEH Amide column $(2.1 \mathrm{~mm} \times 100 \mathrm{~mm}, 1.7 \mu \mathrm{m} ; 0.3 \mathrm{~mL} / \mathrm{min}$ flow rate). For this separation, the mobile phases used were $0.1 \%$ and $0.2 \%$ formic acid (A and B, respectively). A linear gradient was used for separation as follows: $0-0.5 \mathrm{~min}, 15 \% \mathrm{~B}$; 0.5-5.5 min, $15-20 \%$ B; 5.5-12.5 min, $20-40 \%$ B; $12.5-$ $13 \mathrm{~min}, 40-15 \% \mathrm{~B}$; and $13-15 \mathrm{~min}, 15 \% \mathrm{~B}$. An initial $5 \mu \mathrm{L}$ injection volume was used, and the column was warmed to $40{ }^{\circ} \mathrm{C}$. Between each sample injection, the column was washed once twice using a weak $90 \%$ acetonitrile solution and a strong $10 \%$ acetonitrile solution.

A Waters ACQUITY UPLC BEH C8 column $(2.1 \times 100 \mu \mathrm{mm}, 1.7 \mu \mathrm{m} ; 0.26 \mathrm{~mL} / \mathrm{min}$ flow rate) was used for analyses of fatty acids and lipids. For these separations, $60 \%$ acetonitrile in water containing $5 \mathrm{mM}$ ammonium formate and 90\% isopropanol in acetonitrile containing $5 \mathrm{mM}$ ammonium formate served as the mobile phases ( $\mathrm{A}$ and $\mathrm{B}$, respectively). A linear gradient was used for separation as follows: $0-1.0 \mathrm{~min}$, $100 \% \mathrm{~A} ; 1.0-2.0 \mathrm{~min}, 100-70 \% \mathrm{~A} ; 2.0-12.0 \mathrm{~min}, 70-30 \%$ A; $12.0-12.5 \mathrm{~min}, 30-5 \%$ A; $12.5-13.0 \mathrm{~min}, 5-0 \% \mathrm{~A}$; 13.0-14.0 $\mathrm{min}, 0 \% \mathrm{~A} ; 14.0-14.1 \mathrm{~min}, 0-100 \% \mathrm{~A}$ and 13-15 min, $100 \% \mathrm{~A}$. Injection volumes in positive and negative ion mode were $1 \mu \mathrm{L}$ and $2 \mu \mathrm{L}$, respectively, and the column was warmed to $55^{\circ} \mathrm{C}$.

Positive and negative ion modes and multiple reaction monitoring (MRM) were employed during micro mass spectrometer (Xevo TQ-S) operation, with data being acquired and processed using the Waters TargetLynx software.

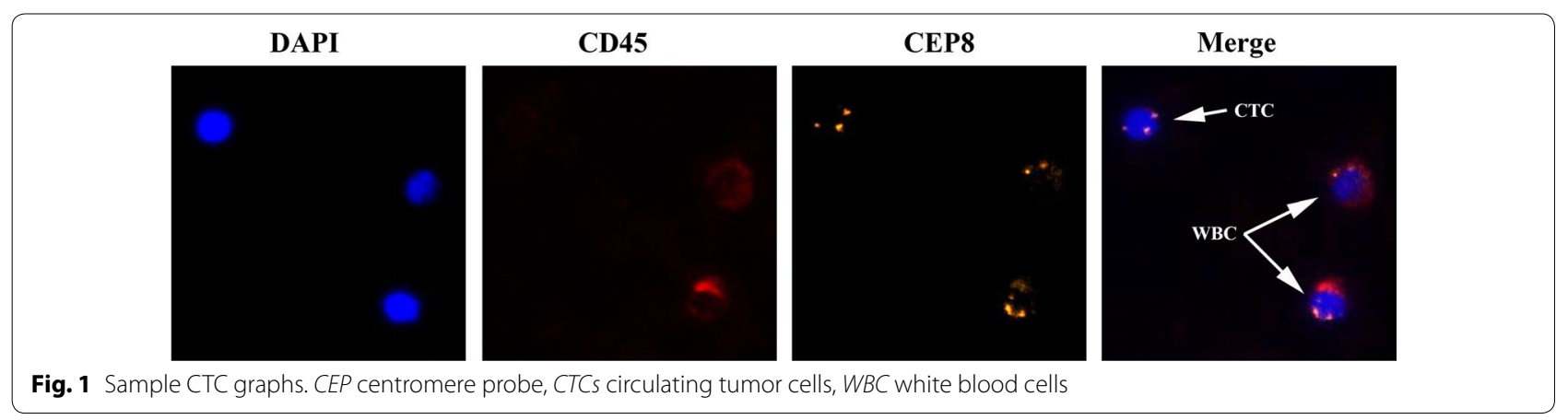




\section{Data analysis}

SPSS (v17, SPSS Inc, IL, USA) was used for all statistical testing. Receiver operating characteristic (ROC) curves were used to explore the diagnostic sensitivity and specificity of CTCs for LC diagnosis. Data were compared via non-parametric Wilcoxon's test and Kruskal-Wallis $\mathrm{H}$ test.

Metaboanalyst [28, 29] was used for multivariate statistical analyses of metabolomics data, including partial least squares discriminant analysis (PLS-DA), biomarker analyses, and pathway analyses. The $R^{2}$ and $Q^{2}$ statistics were utilized to assess PLS-DA model quality, while its reliability was assessed via a permutation test owing to the potential for this model to overestimate separation performance [28, 30]. Univariate analysis, including Student's t-test and Analysis of Variance (ANOVA), were used in the study. Binary logistic regression was carried out for variable selection in the model.

For a pathway analysis of differentially expressed metabolites, pathway modules and pathway topology analyses were used to best identify key biological pathways linked to the observed changes in metabolite levels. The resultant data show both P-values in the pathway enrichment analysis (y-axis) and the pathway impact values from pathway topology analysis ( $\mathrm{x}$-axis), with red being used to identify the most affected pathways.

\section{Results}

\section{CTC detection in LC and control patients}

We have previously used an NE-FISH-based approach to detect CTCs in lung cancer patient peripheral blood samples. In this study, we detected $\geq 1$ CTC in $34 / 51$ (66.67\%) patients prior to surgery (Table 1). Total CTC numbers were from 1 to $30($ mean $=2.57)$, with significantly more CTCs being present in LC patients relative to controls $(P<0.001$, Fig. $2 a)$. An ROC curve was employed to assess the diagnostic utility of CTCs in LC, revealing them to have sensitivity and specificity values of $66.67 \%$ and $92.16 \%$, respectively. This analysis revealed that a cut-off of $0.5 \mathrm{CTCs} / 3.2 \mathrm{~mL}$ of blood was sufficient for LC diagnosis (AUC $=0.812$, 95\% CI $0.722-0.883, P<0.0001$ ) (Fig. 2b). As such, we utilized a 1 CTC cut-off value for LC diagnosis. The CTC positivity rates in SCLC patients were $75 \%$, while in NSCLC patients with adenocarcinomas and squamous cell carcinomas they were $61.29 \%$ and $75 \%$, respectively. TNM stages and lymph node status differed significantly as a function of CTC counts, with all patients with lymph node metastasis having detectable CTCs (Table 1).

After surgery, CTCs were detectable in 34/51 LC patients, with CTC number haven risen in 18 patients, fallen in 21 patients, and remained unchanged in 13 patients.

\section{Targeted metabolomics analyses}

We next explored the levels of specific lipids, fatty acids, and amino acids in LC patient serum through a targeted metabolomics analysis. During the analytical process, quality control samples $(\mathrm{QC})$ were regularly run to ensure result reproducibility, with peak area RSD for these QC samples being < $15 \%$ consistent with good stability and reproducibility. A total of 34 underivatized amino acids were assessed in patient and control serum samples [31]. Direct infusion mode was used for sample introduction into the mass spectrometer. Additional file 1: Table S1 compiles information pertaining to the precursor ions, product ions, and collisions for lipid and fatty acid samples. In total, 24 fatty acids and 147 lipids were detected in this analysis.
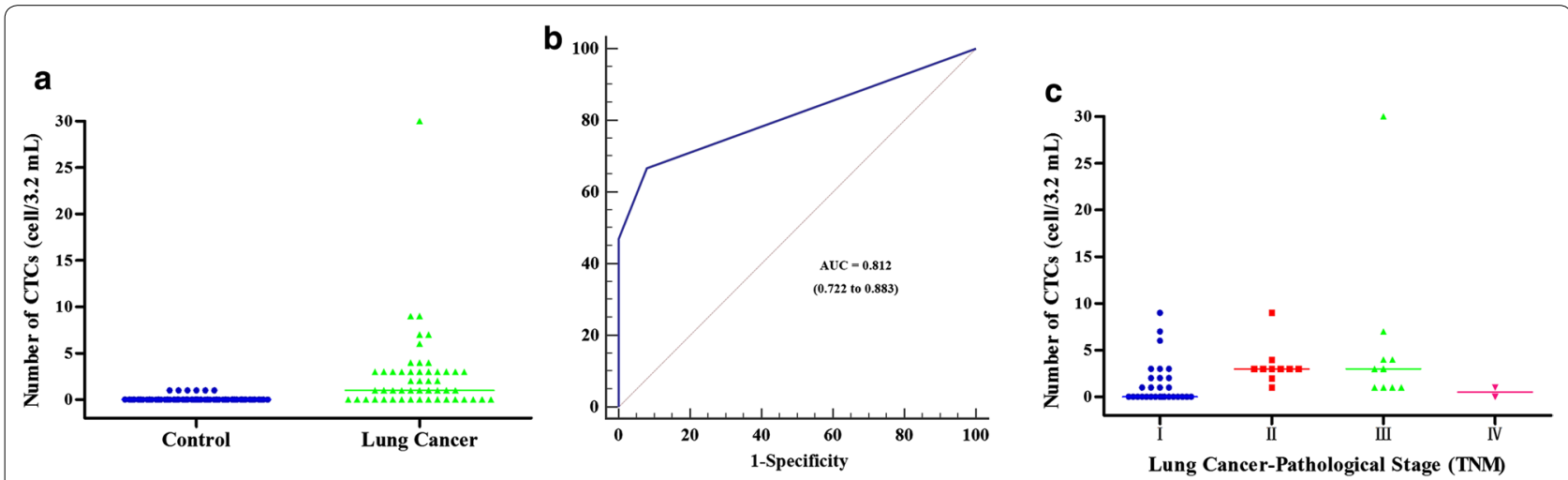

Fig. 2 CTC numbers in lung cancer patients and controls. a CTC distribution in controls and lung cancer patients. b ROC curves used for CTC cutoff value determinations. c CTC distributions in patients with different pathological stages of disease 


\section{Profiling of global metabolic changes in LC patients following surgical intervention}

We employed a PLS-DA approach in order to explore the metabolic differences between the control, LA (LC patients after surgery), and LB (LC patients before surgery) groups (Fig. 3). A permutation test revealed that the $\mathrm{B} / \mathrm{W}$ ratio of the original classes (red arrow) different significantly from the permuted data distribution, consistent with reliable cross-validation $(P<0.05$, Additional file 1: Figure S1). This PLS-DA approach achieved satisfactory classification. Control patients exhibited a significantly distinct metabolic profile, whereas that of the LA and LB groups overlapped slightly (Fig. 3a). However, good separation was achieved between these three groups (Fig. 3b-d). Pre-and post-surgical LA, LB, and control groups could be distinguished on the basis of amino acids and lipid profiles, consisting with significant surgery-related metabolic shifts being present in LC patients and controls (Fig. 3b, d), and there were additional changes between groups LA and LB as a result of surgery.

\section{Differentially abundant metabolite patterns}

A univariate statistical analysis revealed significant changes in lipids, fatty acids and amino acids between the LA, LB, and control groups (Table 2). When comparing the control and LB groups, levels of Cer, PC, SM lipids were higher in the LB group, while levels of LPC, LPE, and TAG were higher in controls. Similarly, LB group samples exhibited higher levels of the amino acids Asn, Lys, Met, Orn, Ser, and Tau relative to controls, whereas Ala, Cys, Gln, and Sar levels were higher in controls. ROC curves were then used to assess the potential value of these metabolites as LC biomarkers in Metaboanalyst [28] (Fig. 3) between LC patients with early-stage (stage I/II) and controls. To avoid overfitting, features were selected based upon overall ranks (AUC and T-statistic) and K-means (KM) clustering. This approach identified SM 42:4, Ser, Sar, Gln, and LPC 18:0 as a potential biomarker panel with an AUC of 0.965 (95\% CI 0.900-1.000) (Additional file 1: Figure S2). As such, this analysis suggests this subset of metabolites may be useful for earlystage LC detection.
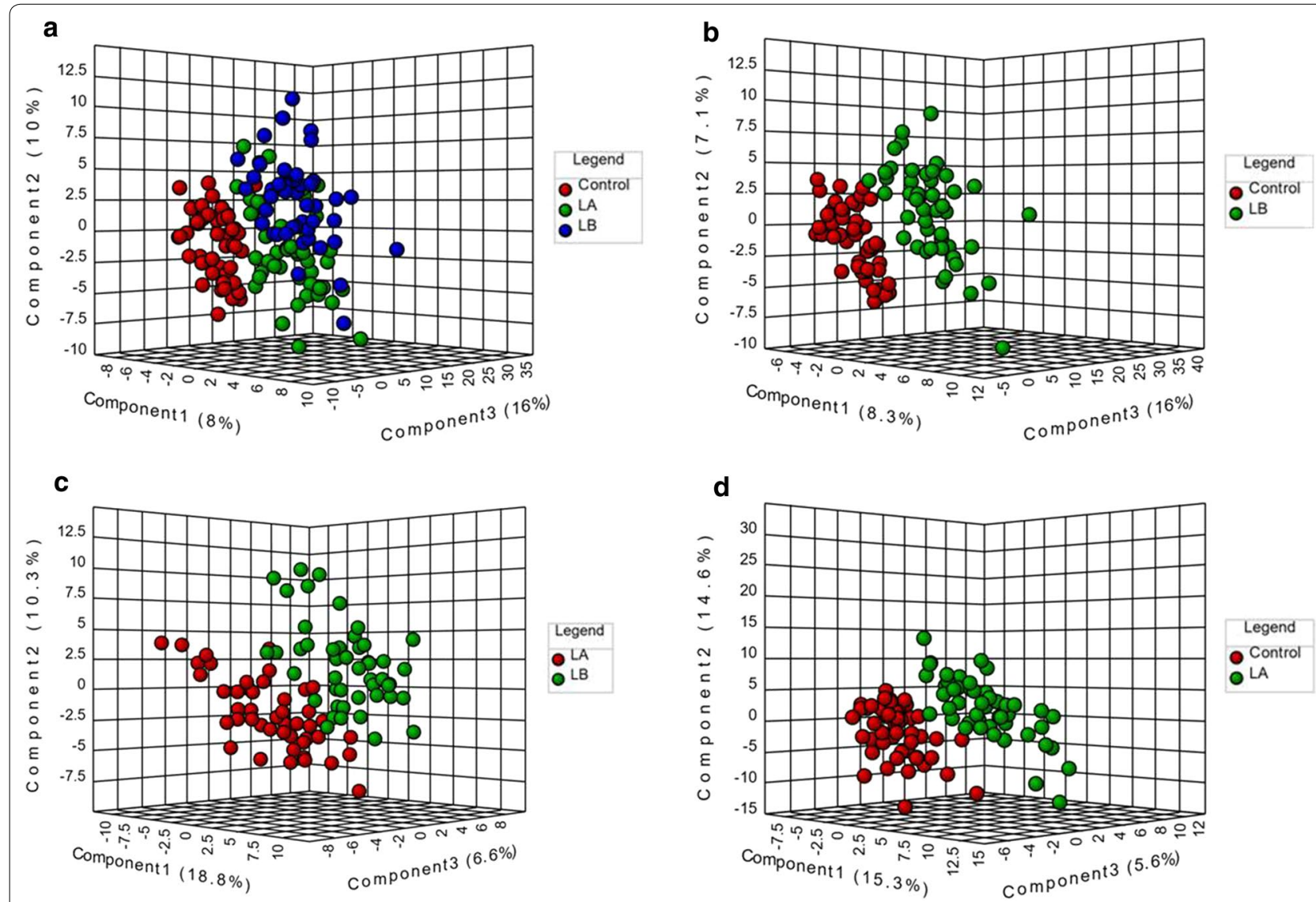

Fig. 3 Score plots for PLS-DA analysis model of metabolomics data the control, LA, and LB groups. a PLS-DA results in all groups; $\mathbf{b}$ PLS-DA results in control and LB groups; $\mathbf{c}$ PLS-DA results in the LA and LB groups; $\mathbf{d}$ PLS-DA results in control and LA groups 
Table 2 Significantly altered metabolites in the different groups

\begin{tabular}{|c|c|c|c|c|}
\hline No. & Metabolites & LB vs control & LA vs control & LB vs LA \\
\hline 1 & Ala & $\# \downarrow$ & $\triangle \downarrow$ & - \\
\hline 2 & Arg & - & $\triangle \uparrow$ & - \\
\hline 3 & Asn & $\# \uparrow$ & - & $\triangle \uparrow$ \\
\hline 4 & Cer 38:1 & $\triangle \uparrow$ & $\triangle \uparrow$ & - \\
\hline 5 & Cer 42:2 & $\triangle \uparrow$ & $\triangle \uparrow$ & - \\
\hline 6 & Cer 43:1 & $\triangle \uparrow$ & $\triangle \uparrow$ & - \\
\hline 7 & Cer d18:1-22:2 & - & $\# \uparrow$ & - \\
\hline 8 & Cit & - & - & $\# \uparrow$ \\
\hline 9 & Cys & $\# \downarrow$ & - & - \\
\hline 10 & FA 20:5 & $\# \downarrow$ & $\# \downarrow$ & - \\
\hline 11 & FA 22:4 & - & $\triangle \uparrow$ & - \\
\hline 12 & FA 22:6 & $\triangle \downarrow$ & - & - \\
\hline 13 & FA 26:0 & $\# \uparrow$ & - & - \\
\hline 14 & GABA & - & $\# \downarrow$ & $\triangle \uparrow$ \\
\hline 15 & Gln & $\triangle \downarrow$ & $\triangle \downarrow$ & - \\
\hline 16 & Ile & - & $\triangle \downarrow$ & - \\
\hline 17 & Leu & - & $\triangle \downarrow$ & - \\
\hline 18 & LPC 14:0 & $\triangle \downarrow$ & $\triangle \downarrow$ & - \\
\hline 19 & LPC 15:0 & $\triangle \downarrow$ & $\# \downarrow$ & - \\
\hline 20 & LPC 16:0 & $\triangle \downarrow$ & $\triangle \downarrow$ & - \\
\hline 21 & LPC 16:1 & $\triangle \downarrow$ & $\# \downarrow$ & - \\
\hline 22 & LPC 17:0 & $\triangle \downarrow$ & $\triangle \downarrow$ & - \\
\hline 23 & LPC 17:1 & $\triangle \downarrow$ & $\# \downarrow$ & - \\
\hline 24 & LPC 18:0 & $\triangle \downarrow$ & $\triangle \downarrow$ & - \\
\hline 25 & LPC 18:1 & $\triangle \downarrow$ & $\# \downarrow$ & - \\
\hline 26 & LPC 20:0 & $\triangle \downarrow$ & $\triangle \downarrow$ & - \\
\hline 27 & LPC 20:1 & $\triangle \downarrow$ & $\triangle \downarrow$ & - \\
\hline 28 & LPC 20:2 & - & $\triangle \downarrow$ & $\triangle \uparrow$ \\
\hline 29 & LPC 22:0 & - & $\# \downarrow$ & - \\
\hline 30 & LPC 22:4 & - & $\# \uparrow$ & - \\
\hline 31 & LPC 22:5 & - & $\# \uparrow$ & - \\
\hline 32 & LPC 22:6 & $\# \downarrow$ & - & - \\
\hline 33 & LPC18:4 & $\triangle \downarrow$ & $\triangle \downarrow$ & - \\
\hline 34 & LPC 22:5 & - & $\# \uparrow$ & - \\
\hline 35 & LPC 22:6 & $\# \downarrow$ & - & - \\
\hline 36 & LPC18:4 & $\triangle \downarrow$ & $\triangle \downarrow$ & - \\
\hline 37 & LPE 16:0 & $\# \downarrow$ & - & - \\
\hline 38 & LPE 20:2 & - & $\# \downarrow$ & $\triangle \uparrow$ \\
\hline 39 & LPE 20:4 & - & $\triangle \uparrow$ & - \\
\hline 40 & LPE 20:5 & $\triangle \downarrow$ & $\triangle \downarrow$ & - \\
\hline 41 & Lys & $\# \uparrow$ & $\triangle \uparrow$ & - \\
\hline 42 & Met & $\triangle \uparrow$ & - & $\# \uparrow$ \\
\hline 43 & Orn & $\triangle \uparrow$ & $\# \uparrow$ & - \\
\hline 44 & PC 29:1 & - & - & $\# \downarrow$ \\
\hline 45 & PC 30:0 & $\# \uparrow$ & $\triangle \uparrow$ & - \\
\hline 46 & PC 30:1 & $\triangle \uparrow$ & $\triangle \uparrow$ & - \\
\hline 47 & PC 31:0 & - & $\# \uparrow$ & - \\
\hline 48 & PC 32:0 & $\triangle \uparrow$ & $\triangle \uparrow$ & - \\
\hline 49 & PC 32:1 & - & $\triangle \uparrow$ & - \\
\hline
\end{tabular}

Table 2 (continued)

\begin{tabular}{|c|c|c|c|c|}
\hline No. & Metabolites & LB vs control & LA vs control & LB vs LA \\
\hline 50 & PC 33:1 & - & $\# \uparrow$ & - \\
\hline 51 & PC 33:2 & - & $\# \uparrow$ & - \\
\hline 52 & PC 34:0 & - & $\triangle \uparrow$ & - \\
\hline 53 & PC 34:3 & $\# \uparrow$ & $\triangle \uparrow$ & - \\
\hline 54 & PC 34:4 & - & $\# \uparrow$ & - \\
\hline 55 & PC 34:5 & - & $\# \uparrow$ & - \\
\hline 56 & PC 35:1 & $\# \uparrow$ & $\triangle \uparrow$ & - \\
\hline 57 & PC 35:3 & $\# \uparrow$ & - & - \\
\hline 58 & PC 36:0 & $\triangle \uparrow$ & - & - \\
\hline 59 & PC 37:3 & $\# \uparrow$ & - & - \\
\hline 60 & PC 37:4 & $\# \uparrow$ & - & - \\
\hline 61 & PC 38:2 & $\# \uparrow$ & - & - \\
\hline 62 & PC 38:5 & $\triangle \uparrow$ & - & - \\
\hline 63 & PC 40:4 & $\triangle \uparrow$ & $\triangle \uparrow$ & \\
\hline 64 & PC 40:8 & $\# \uparrow$ & $\triangle \uparrow$ & - \\
\hline 65 & PC 42:2 & - & $\# \downarrow$ & - \\
\hline 66 & PC 42:4 & - & $\# \downarrow$ & - \\
\hline 67 & PC 42:5 & - & $\triangle \uparrow$ & - \\
\hline 68 & PC 42:8 & $\# \uparrow$ & - & - \\
\hline 69 & PC 43:5 & $\triangle \uparrow$ & $\triangle \uparrow$ & - \\
\hline 70 & PC33:3 & - & $\# \uparrow$ & - \\
\hline 71 & PC38:0 & - & $\triangle \uparrow$ & - \\
\hline 72 & PE16:0-16:0 & $\# \uparrow$ & $\# \uparrow$ & - \\
\hline 73 & Phe & - & $\triangle \downarrow$ & - \\
\hline 74 & Sar & $\Delta \downarrow$ & $\Delta \downarrow$ & - \\
\hline 75 & Ser & $\triangle \uparrow$ & - & $\# \uparrow$ \\
\hline 76 & SM 31:1 & $\# \downarrow$ & - & - \\
\hline 77 & SM 32:2 & - & $\# \uparrow$ & - \\
\hline 78 & SM 34:0 & $\triangle \uparrow$ & - & - \\
\hline 79 & SM 34:1 & $\# \uparrow$ & $\triangle \uparrow$ & - \\
\hline 80 & SM 34:2 & $\triangle \uparrow$ & $\triangle \uparrow$ & - \\
\hline 81 & SM 35:0 & $\# \uparrow$ & $\triangle \uparrow$ & $\Delta \downarrow$ \\
\hline 82 & SM 35:1 & $\triangle \uparrow$ & $\triangle \uparrow$ & - \\
\hline 83 & SM 36:1 & $\triangle \uparrow$ & $\triangle \uparrow$ & - \\
\hline 84 & SM 36:2 & $\triangle \uparrow$ & $\triangle \uparrow$ & - \\
\hline 85 & SM 36:3 & - & $\triangle \uparrow$ & - \\
\hline 86 & SM 37:1 & - & $\triangle \uparrow$ & $\triangle \downarrow$ \\
\hline 87 & SM 37:2 & - & $\# \uparrow$ & - \\
\hline 88 & SM $38: 3$ & - & $\triangle \uparrow$ & - \\
\hline 89 & SM 41:1 & - & $\triangle \uparrow$ & - \\
\hline 90 & SM 42:2 & $\triangle \uparrow$ & $\triangle \uparrow$ & - \\
\hline 91 & SM 42:4 & $\triangle \uparrow$ & $\triangle \uparrow$ & - \\
\hline 92 & SM 42:6 & - & $\triangle \uparrow$ & - \\
\hline 93 & SM 43:1 & $\# \downarrow$ & - & - \\
\hline 94 & SM 43:2 & $\# \uparrow$ & $\triangle \uparrow$ & - \\
\hline 95 & SM 35:3 & $\triangle \uparrow$ & $\triangle \uparrow$ & - \\
\hline 96 & TAG 50:0 & - & $\# \uparrow$ & $\Delta \downarrow$ \\
\hline 97 & TAG 52:3 & $\# \downarrow$ & - & - \\
\hline 98 & TAG 54:1 & $\# \downarrow$ & - & - \\
\hline
\end{tabular}


Table 2 (continued)

\begin{tabular}{lllll}
\hline No. & Metabolites & LB vs control & LA vs control & LB vs LA \\
\hline 99 & TAG 54:2 & $\triangle \downarrow$ & $\# \downarrow$ & - \\
100 & TAG 54:3 & $\# \downarrow$ & - & - \\
101 & TAG 54:4 & $\# \downarrow$ & - & - \\
102 & Tau & $\# \uparrow$ & $\# \uparrow$ & - \\
103 & Thr & - & $\triangle \downarrow$ & $\triangle \uparrow$ \\
104 & Val & - & $\# \downarrow$ & $\triangle \uparrow$ \\
\hline
\end{tabular}

Following surgery, lower levels of Ala, GABA, Gln, Ile, Leu, Phe, Sar, Thr, Val, LPC, and LPE were detected in samples from the LA group relative to controls, whereas levels of Arg, Orn, Tau, Cer, SM, and PC were higher in the LA group relative to controls. Furthermore, significant changes in the levels of Asn, Cit, GABA, LPC 20:2, LPE 20:2, Met, PC 29:1, Ser, SM 35:0, SM 37:1, TAG 50:0, Thr, and SM 37:1 were detected when comparing preand post-surgery samples (Table 2).

\section{Metabolic pathway analysis}

We next explored the potential pathways involved in regulating the differentially abundant metabolites detected in the above analysis using MetaboAnalyst, with those pathways that had low $P$-values and high pathway impacts being shown in Fig. 4.

Prior to surgery, LC-specific metabolites were primarily associated with the following metabolic pathways: Glycine, serine and threonine metabolism, Sphingolipid metabolism, Glycerophospholipid metabolism, Taurine and hypotaurine metabolism, Alanine, aspartate and glutamate metabolism, Arginine biosynthesis, Arginine and proline metabolism, Aminoacyl-tRNA biosynthesis, and Glyoxylate and dicarboxylate metabolism (Fig. 4a).

Following surgery, metabolites were primarily associated with: glycine, serine and threonine metabolism, alanine, aspartate and glutamate metabolism, sphingolipid metabolism, arginine biosynthesis, arginine and proline metabolism, arachidonic acid metabolism, aminoacyltRNA biosynthesis, and glyoxylate and dicarboxylate metabolism (Fig. 4b). In addition, 11 metabolic pathways were identified when comparing post-surgery LC patients to controls (Fig. 4c). For full details of affected pathways, see Additional file 1: Table S2.

\section{The prognostic relevance of CTCs and metabolic signatures in LC}

Upon follow-up, 14 patients exhibited recurrent disease (SD + PD), with CTC numbers being increased in 13 of these patients following surgery. In contrast, 31 patients with non-recurrent disease (PR + CR), with CTC numbers being reduced in 29 of these patients following surgery (Additional file 1: Table S3). This suggests that CTC trends following surgery are consistent with patient prognosis.

Among the metabolites related to relapse, 25 metabolites were found to be significant increased in recurrent patients after surgery compared with nonrecurrent patients and the controls based on ANOVA analysis. Binary logistic regression was introduced for the early prediction of LC recurrence to analyze 25 metabolites. With respect to these metabolites, levels of SM 42:2, SM 35:1, PC 30:0, PC 30:1, Cer 42:2, and SM 38:3 were found to be present at significantly higher levels post-surgery in patients that suffered from LC recurrence relative to patients with non-recurrent disease or controls(Fig. 5)
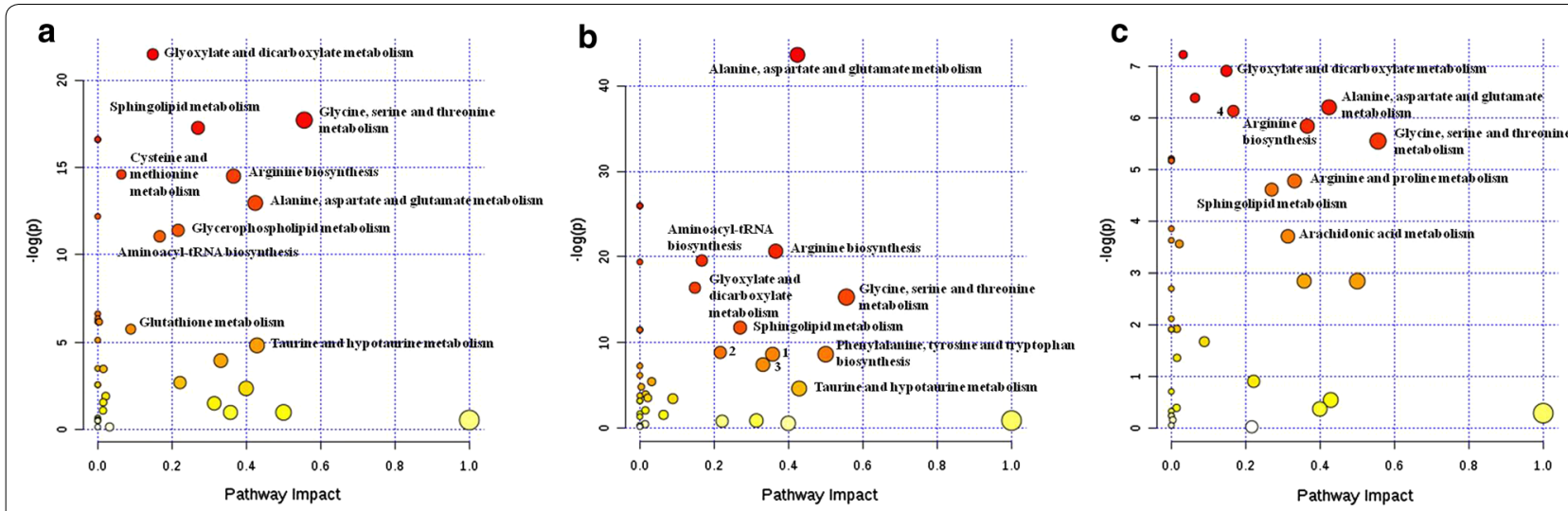

Fig. 4 Significant differences in specific metabolic pathways were detected between the control, LA, and LB groups. Node size corresponds to the degree of impact on the indicated pathway, while node color corresponds to the associated $P$-value derived from a pathway enrichment analysis. a LB vs control; b LA vs control; c LB vs LA. 1: phenylalanine metabolism; 2: glycerophospholipid metabolism; 3: arginine and proline metabolism; 4: aminoacyl-tRNA biosynthesis 

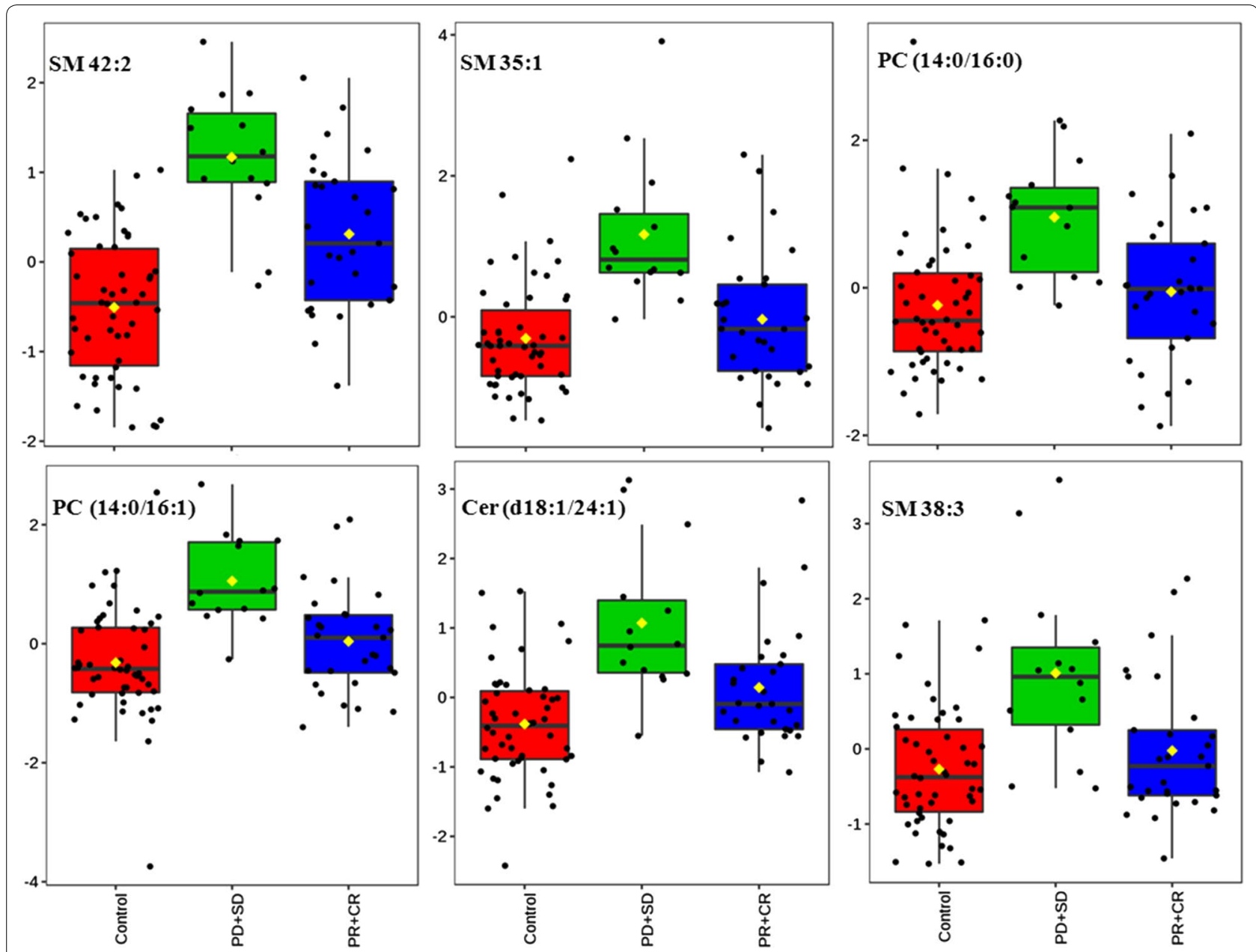

Fig. 5 Box plots corresponding to levels of the six indicated metabolites in controls, patients with non-recurrent disease, and patients with recurrent disease. Non-recurrent patients: PR + CR group; recurrent patients: SD + PD group

and showed significant changes $(P<0.05)$ from binary logistic regression. This therefore suggests that this metabolite panel may offer prognostic value as a means of predicting the likelihood of LC recurrence in patients undergoing surgery.

\section{Discussion}

In the present study, we explored the relationship between CTCs, metabolic signatures, and LC patient post-surgical outcomes. We were able to detect CTCs in $66.7 \%$ of analyzed patients $(34 / 51)$ prior to surgery. We observed surgery-related changes in CTC levels consistent with observed clinical outcomes (RECIST standard). Previous work suggests that CTCs may be valuable as diagnostic biomarkers of $\mathrm{LC}$ or for the identification of patients likely to benefit from specific treatments [32, 33]. Owing to the rarity of these cells and their non-epithelial characteristics, however, detecting CTCs in LC patients remains challenging. The previously reported
CTC detection approach employed in the present study was nonetheless able to offer satisfactory clinical utility in LC [26].

Early LC diagnosis is essential, as patients with earlystage LC have a much better prognosis relative to patients with advanced disease [34, 35]. The identification of additional sensitive and specific biomarkers of LC is essential to facilitate the better detection and management of early-stage disease. Certain lipid signatures and other metabolites have been reported to be well-suited to differentiating between LC patients and healthy controls or individuals with non-malignant lung disease [36-39]. Metabolites in these signatures included choline-containing phospholipids, with alterations in precursor serum lysophosphatidylcholine levels thus having the potential to serve as valuable cancer biomarkers given that the metabolism of phospholipids such as phosphatidylcholine can be significantly disrupted in tumor cells [40]. We identified 14 LPC classes that were decreased in LC 
patient serum relative to controls, in line with previous research [38, 41, 42]. LPC acyltransferases (LPCATs) can convert LPC to PC in lung surfactant, which can lower surface tension and thereby facilitate respiration. LPCAT dysfunction is a hallmark of lung diseases, thus resulting in significant changes in LPC levels in affected individuals. Interestingly, we found that relative to controls, the levels of most measured PC classes were increased in LC patients prior to surgery. Chen et al. [43] detected significantly elevated levels of both monounsaturated and saturated PCs in LC patients, whereas these patients had lower polyunsaturated PC levels. Yet another study found that PC levels were elevated in LC patient serum [44]. Such elevated PC levels may be due to an increased need for these membrane phospholipids in cancer cells, given that they are primary components of the cell membrane $[45,46]$.

We observed elevated levels of sphingolipids such as ceramide (Cer) and sphingomyelin (SM) in LC patients relative to controls both pre- and post-surgery (Table 2 ). Sphingolipids are key bioactive molecules that can facilitate intracellular signaling and can control the growth and survival of tumor cells [47]. Cer is also thought to function as a pro-apoptotic lipid, the levels of which are increased in response to stress, thereby suppressing tumor growth [48]. Both chemotherapy and cytotoxic stimulation can promote endogenous Cer accumulation. Reduced amounts of Cer have been detected in both human colon cancer tissue [49] and ovarian tumors [50]. Despite these findings, other analyses have revealed that certain Cer species are in fact upregulated in cancer. For example, higher levels of long-chain ceramides have been detected in malignant breast cancer tissues relative to benign tissue controls [51]. This is consistent with our findings, suggesting that certain Cer species play specific roles in tumors. SM is a primary sphingolipid type found in cell membranes, and it can be converted into Cer by sphingomyelinase. Studies have explored the chemotherapeutic and chemopreventive potential of this pathway [52]. In one study, sphingomyelin hydrolysis and consequent disruption of ceramide synthesis were found to be associated with reduced NSCLC growth and enhanced anti-tumor immunity [53, 54].

We employed a rapid approach to sensitively and specifically detecting and quantifying the levels of 34 amino acids without derivatization in this study. Amino acids play central roles in metabolic networks, and have previously been shown to be of value for discriminating between LC patients and healthy controls [55-58]. The amino acid signatures detected in the present study were not identical to those in prior studies, potentially due to differences in population ethnicity, tumor stages, or LC histologic types in these studies [55-58]. However, the differentially abundant amino acid levels identified in this study have the potential to be used for early-stage LC diagnosis.

Surgery remains the best treatment option for LC patients with early-stage (stage I/II) disease, as it is associated with a significant increase in long-term patient survival $[4,5]$. In a PLS-DA analysis, we found that metabolic shifts were evident in LC patients and controls (Fig. 3). We further employed pathway analyses in an effort to identify LC-related signaling pathways associated with the observed metabolic changes in our samples. These analyses revealed that these differentially abundant metabolites were associated with host responses to LC (Fig. 4a). In total, 9 and 11 differentially impacted metabolic pathways were identified in LC patients relative to controls before and after surgery, respectively (Fig. 4). A total of 8 metabolic pathways in LC patients with impacted by surgical intervention (Fig. $4 \mathrm{~b}$ ). The most significantly affected of these pathways included, the glycine, serine, and threonine metabolism pathways. Serine can be utilized for the synthesis of intermediates in the glycine, serine and threonine metabolism, protein, sphingolipid, purine and pyrimidine pathways [59]. Increased serine biosynthesis has been shown to drive cancer cell proliferation and to increase transformation rates in normal mammary epithelial cells [60]. Serine synthesis reportedly accounts for roughly half of total TCA cycle replenishment [59]. The upregulation of intermediates in the glycine, serine, and threonine metabolism pathways may thus be strongly correlated with increased glycolysis and of TCA cycle intermediate replenishment. In addition, we found that other amino acid-related pathways were associated with surgery in LC patients, including the Arginine biosynthesis, alanine, aspartate and glutamate metabolism, and Arginine and proline metabolism pathways.

We identified six different metabolites that were present at significantly higher levels in patients that went on to suffer from $\mathrm{LC}$ recurrence relative to patients with non-recurrent disease, including SM 42:2, SM 35:1, PC (16:0/14:0), PC (14:0/16:1), Cer (d18:1/24:1), and SM 38:3 (SD $+\mathrm{PD}$ group vs. $\mathrm{PR}+\mathrm{CR}$ group, $P<0.05)$. This suggests that this biomarker combination may be of value for LC diagnosis and or prediction of LC recurrence in patients undergoing surgery (Fig. 5).

There are several limitations to the present analysis that must be considered. For one, this study was of a relatively small LC patient population, and future large-scale studies will be essential to draw any definitive conclusions regarding metabolic profiles associated with LC patient outcomes. In addition, additional assays are necessary to more fully explore and validate the surgery-associated metabolic changes detected in this study in LC patients, 
offering more insight into surgery-related pathophysiology. In addition, at present no correlation has been detected between CTCs and metabolic signatures. Future studies conducting single-cell metabolic profiling of these CTCs have the potential to offer direct functional insights into tumor cell metabolism in individual patients, facilitating a more direct understanding of the link between cancer cell genotype and metabolomics phenotype [61].

\section{Conclusions}

In summary, our study demonstrates that LC patients had significantly higher CTC counts relative to healthy controls and changes of CTC counts were associated with clinical outcomes following surgery. Moreover, six metabolites were defined as the combinational biomarkers to recognize recurrence and nonrecurrence based on target metabolomics. Based on these essential findings, we suggest that CTC detection and plasma metabolite profiling may be an effective means of diagnosing early-stage LC and identifying patients at risk for disease recurrence.

\section{Supplementary information} org/10.1186/s12967-020-02401-0.

Additional file 1: Table S1. All the annotated lipids in current study. Table S2. List of all the changed pathways among three groups based on metabolic pathway analysis. Table S3. Patients information and their prognosis status. Figure S1. Validation of PLS-DA model using permutation test. Figure S2. ROC curves for five metabolites (SM 42:4, Ser, Sar, GIn and LPC 18:0) to discriminate lung cancer patients from controls.
Supplementary information accompanies this paper at https://doi.

target metabolomics analysis. YL, RF and PH performed CTC detection. PZ, TR and YW provided clinical samples and clinical information. NG and HY provided advice and supervised. All authors read and approved the final manuscript.

\section{Funding}

This work was financially supported by the Fundamental Research Funds for the central public welfare research institutes (Project number zz2017004, GH201916) and the National Major Science and Technology Projects of China (81370095), the National Science and Technology Major Project (grant number 2017ZX09301040), State Key Laboratory of Generic Manufacture Technology of Traditional Chinese Medicine and Shandong Natural Science Foundation of China (ZR2017LH050 and ZR2019PH084).

\section{Availability of data and materials}

All data generated or analyzed during this study are included in this article and its additional files.

\section{Ethics approval and consent to participate}

This study was approved by the Ethics Committee of Liaocheng People's Hospital. All patients were fully informed of the experimental procedures before sample acquisition.

\section{Consent for publication}

Not applicable.

\section{Competing interests}

The authors declare that they have no competing interests.

\section{Author details}

1 Zhong Yuan Academy of Biological Medicine, Liaocheng People's Hospital, Liaocheng 252000, People's Republic of China. ${ }^{2}$ Experimental Research Center, China Academy of Chinese Medical Sciences, Beijing 100700, People's Republic of China. ${ }^{3}$ Department of Respiratory Medicine, Liaocheng People's Hospital, Liaocheng 252000, People's Republic of China. ${ }^{4}$ Institute of Chinese Materia Medica, China Academy of Chinese Medical Sciences, Beijing 100700, People's Republic of China. ${ }^{5}$ State Key Laboratory of Generic Manufacture Technology of Traditional Chinese Medicine, Lunan Pharmaceutical Group Co. Ltd., Shandong 276006, People's Republic of China.

Received: 10 March 2020 Accepted: 3 June 2020

Published online: 17 June 2020

\section{Abbreviations}

LC: Lung cancer; NE-FISH: Negative enrichment-fluorescence in situ hybridization; CTCs: Circulating tumor cells; ctDNA: Circulating tumor DNA; CEA: Carcinoembryonic antigen; AFP: Alpha-fetoprotein; NSCLC: Non-small cell lung cancer; SCLC: Small cell lung cancer; UPLC/MS: Ultra-performance liquid chromatography/mass spectrometry; UPLC-QTOF/MS: Ultra-performance liquid chromatography with quadrupole time-of-flight mass spectrometry; Gly: Glycine; $\beta$-Ala: $\beta$-Alanine; Sar: Sarcosine; Ala: L-Alanine; Ser: L-Serine; Pro: L-Proline; Val: L-Valine; Thr: L-Threonine; Cys: L-Cysteine; Tau: Taurine; Leu: L-Leucine; GABA: Y-Aminobutyric acid; Ile: L-Isoleucine; t-Hyp: Trans-4-hydroxyL-proline; Asn: L-Asparagine; Asp: L-Aspartic acid; Orn: L-Ornithine; Lys: L-Lysine; Glu: L-Glutamic acid; Met: L-Methionine; Phe: L-Phenylalanine; Arg: L-Arginine; Cit: L-Citrulline; Tyr: L-Tyrosine; Trp: L-Tryptophan; FA: Folic acid; His: L-Histidine; Gln: Glutamine; LPC: Lysophosphatidylcholine; PC: Phosphatidylcholine; LPE: Lysophosphatidylethanolamine; PE: Phosphatidylethanolamine; SM: Sphingomyelin; Cer: Ceramide; TAG: Triacylglycerol; IS: Internal standard; RT: Room temperature; CR: Complete response; SD: Stable disease; PR: Partial response; PD: Progressive disease; CEP: Chromosome centromere probe; ROC: Receiver operating characteristic; PLS-DA: Partial least squares discriminant analysis; LA: LC patients after surgery; LB: LC patients before surgery; LPCATs: LPC acyltransferases; RECIST: Response Evaluation Criteria in Solid Tumors.

\section{Acknowledgements}

Not applicable.

\section{Author's contributions}

The project was conceived by NG, HY and DY. DY and NG designed the strategy, interpreted the data and wrote the manuscript. XY and NG performed

\section{References}

1. Bray F, Ferlay J, Soerjomataram I, Siegel RL, Torre LA, Jemal A. Global cancer statistics 2018: GLOBOCAN estimates of incidence and mortality worldwide for 36 cancers in 185 countries. CA Cancer J Clin. 2018:68:394-424

2. Lemjabbar-Alaoui H, Hassan OU, Yang Y-W, Buchanan P. Lung cancer: biology and treatment options. BBA Rev Cancer. 2015;1856:189-210.

3. Spira A, Halmos B, Powell CA. Update in lung cancer 2014. Am J Resp Crit

4. Tomaszek SC, Wigle DA. Surgical management of lung cancer. Semin Resp Crit Care. 2011;32:69-77.

5. Kanwal M, Ding X-J, Cao Y. Familial risk for lung cancer (Review). Oncol Lett. 2017;13:535-42.

6. Lang-Lazdunski L. Surgery for nonsmall cell lung cancer. Eur Respir Rev. 2013;22:382-404

7. Jones GS, Baldwin DR. Recent advances in the management of lung cancer. Clin Med. 2018;18:S41-6.

8. Speicher MR, Pantel K. Tumor signatures in the blood. Nat Biotechnol. 2014;32:441.

9. Pantel K, Speicher M. The biology of circulating tumor cells. Oncogene. 2016;35:1216

10. Alix-Panabières $C$, Pantel K. Challenges in circulating tumour cell research. Nat Rev Cancer. 2014;14:623.

11. Alix-Panabières C, Pantel K. Clinical applications of circulating tumor cells and circulating tumor DNA as liquid biopsy. Cancer Discov. 2016;6:479-91. Care. 2015;192:283-94. 
12. Krebs MG, Hou J-M, Sloane R, Lancashire L, Priest L, Nonaka D, Ward $\mathrm{TH}$, Backen A, Clack G, Hughes A. Analysis of circulating tumor cells in patients with non-small cell lung cancer using epithelial marker-dependent and-independent approaches. J Thorac Oncol. 2012;7:306-15.

13. Huang J, Wang K, Xu J, Huang J, Zhang T. Prognostic significance of circulating tumor cells in non-small-cell lung cancer patients: a meta-analysis. PLOS ONE. 2013;8:e78070.

14. Hou J-M, Krebs MG, Lancashire L, Sloane R, Backen A, Swain RK, Priest L, Greystoke A, Zhou C, Morris K. Clinical significance and molecular characteristics of circulating tumor cells and circulating tumor microemboli in patients with small-cell lung cancer. J Clin Oncol. 2012;30:525-32.

15. Hiltermann T, Pore M, Van den Berg A, Timens W, Boezen H, Liesker J, Schouwink J, Wijnands W, Kerner G, Kruyt F. Circulating tumor cells in small-cell lung cancer: a predictive and prognostic factor. Ann Oncol. 2012;23:2937-42.

16. Ni X, Zhuo M, Su Z, Duan J, Gao Y, Wang Z, Zong C, Bai H, Chapman AR, Zhao J. Reproducible copy number variation patterns among single circulating tumor cells of lung cancer patients. Proc Natl Acad Sci. 2013;110:21083-8.

17. Carter L, Rothwell DG, Mesquita B, Smowton C, Leong HS, FernandezGutierrez F, Li Y, Burt DJ, Antonello J, Morrow CJ. Molecular analysis of circulating tumor cells identifies distinct copy-number profiles in patients with chemosensitive and chemorefractory small-cell lung cancer. Nat Med. 2017;23:114

18. Yeo T, Tan SJ, Lim CL, Lau DPX, Chua YW, Krisna SS, lyer G, San Tan G, Lim $\mathrm{TKH}$, Tan DS. Microfluidic enrichment for the single cell analysis of circulating tumor cells. Sci Rep. 2016;6:22076.

19. Armitage EG, Ciborowski M. Applications of metabolomics in cancer studies. Adv Exp Med Biol. 2017:965:209-34.

20. Burton C, Ma Y. Current trends in cancer biottiarker discovery using urinary metabolomics: achievements and new challenges. Curr Med Chem. 2019;26:5-28.

21. Cheung PK, Ma MH, Tse HF, Yeung KF, Tsang HF, Chu MKM, Kan CM, Cho WCS, Ng LBW, Chan LWC, Wong SCC. The applications of metabolomics in the molecular diagnostics of cancer. Expert Rev Mol Diagn. 2019;19:785-93.

22. Beger RD. A review of applications of metabolomics in cancer. Metabolites. 2013;3:552-74.

23. Bamji-Stocke S, van Berkel V, Miller DM, Frieboes HB. A review of metabolism-associated biomarkers in lung cancer diagnosis and treatment. Metabolomics. 2018;14:81.

24. Wang $X$, Sun $H$, Zhang $A$, Wang $P$, Han $Y$. Ultra-performance liquid chromatography coupled to mass spectrometry as a sensitive and powerful technology for metabolomic studies. J Sep Sci. 2011;34:3451-9.

25. Zhao Y-Y, Wu S-P, Liu S, Zhang Y, Lin R-C. Ultra-performance liquid chromatography-mass spectrometry as a sensitive and powerful technology in lipidomic applications. Chem-Biol Interac. 2014;220:181-92.

26. Li Y, Tian X, Gao L, Jiang X, Fu R, Zhang T, Ren T, Hu P, Wu Y, Zhao P. Clinical significance of circulating tumor cells and tumor markers in the diagnosis of lung cancer. Cancer Med. 2019;8:3782-92.

27. Li Y, Ma G, Zhao P, Fu R, Gao L, Jiang X, Hu P, Ren T, Wu Y, Wang Z. Improvement of sensitive and specific detection of circulating tumor cells using negative enrichment and immunostaining-FISH. Clin Chim Acta. 2018:485:95-102.

28. Chong J, Wishart DS, Xia J. Using metaboanalyst 4.0 for comprehensive and integrative metabolomics data analysis. Curr Protoc Bioinformatics. 2019;68:e86.

29. Chong J, Soufan O, Li C, Caraus I, Li S, Bourque G, Wishart DS, Xia J. MetaboAnalyst 4.0: towards more transparent and integrative metabolomics analysis. Nucleic Acids Res. 2018;46:W486-94.

30. Ye G, Zhu B, Yao Z, Yin P, Lu X, Kong H, Fan F, Jiao B, Xu G. Analysis of urinary metabolic signatures of early hepatocellular carcinoma recurrence after surgical removal using gas chromatography-mass spectrometry. J Proteome Res. 2012;11:4361-72

31. Guo N, Yang D, Yang X, Yan H, Fan B, Dai J, Lei Y, Yan D. A rapid, sensitive, and widely applicable method for quantitative analysis of underivatized amino acids in different biological matrices by UHPLC-MS/MS. J Sep Sci. 2019;42:3173-81.

32. Krebs MG, Metcalf RL, Carter L, Brady G, Blackhall FH, Dive C. Molecular analysis of circulating tumour cells-biology and biomarkers. Nat Rev Clin Oncol. 2014;11:129.
33. Pawlikowska P, Faugeroux V, Oulhen M, Aberlenc A, Tayoun T, Pailler E, Farace F. Circulating tumor cells (CTCS) for the noninvasive monitoring and personalization of non-small cell lung cancer (NSCLC) therapies. J Thorac Dis. 2019;11:S45

34. Dillman RO, McClure SE. Steadily improving survival in lung cancer. Clin Lung Cancer. 2014;15:331-7.

35. Miller KD, Siegel RL, Lin CC, Mariotto AB, Kramer JL, Rowland JH, Stein KD, Alteri R, Jemal A. Cancer treatment and survivorship statistics, 2016. CA Cancer J Clin. 2016:66:271-89.

36. Mazzone PJ, Wang X-F, Beukemann M, Zhang Q, Seeley M, Mohney R, Holt T, Pappan KL. Metabolite profiles of the serum of patients with non-small cell carcinoma. J Thorac Oncol. 2016;11:72-8.

37. Louis E, Adriaensens P, Guedens W, Bigirumurame T, Baeten K, Vanhove K, Vandeurzen K, Darquennes K, Vansteenkiste J, Dooms C. Detection of lung cancer through metabolic changes measured in blood plasma. J Thorac Oncol. 2016;11:516-23.

38. Guo Y, Wang X, Qiu L, Qin X, Liu H, Wang Y, Li F, Wang X, Chen G, Song G. Probing gender-specific lipid metabolites and diagnostic biomarkers for lung cancer using Fourier transform ion cyclotron resonance mass spectrometry. Clin Chim Acta. 2012;414:135-41.

39. Yu Z, Chen H, Ai J, Zhu Y, Li Y, Borgia JA, Yang J-S, Zhang J, Jiang B, Gu W. Global lipidomics identified plasma lipids as novel biomarkers for early detection of lung cancer. Oncotarget. 2017;8:107899.

40. Ackerstaff E, Glunde K, Bhujwalla ZM. Choline phospholipid metabolism: a target in cancer cells? J Cell Biochem. 2003;90:525-33.

41. Ros-Mazurczyk M, Jelonek K, Marczyk M, Binczyk F, Pietrowska M, Polanska J, Dziadziuszko R, Jassem J, Rzyman W, Widlak P. Serum lipid profile discriminates patients with early lung cancer from healthy controls. Lung Cancer. 2017;112:69-74

42. Dong J, Cai X, Zhao L, Xue X, Zou L, Zhang X, Liang X. Lysophosphatidylcholine profiling of plasma: discrimination of isomers and discovery of lung cancer biomarkers. Metabolomics. 2010;6:478-88.

43. Chen Y, Ma Z, Zhong J, Li L, Min L, Xu L, Li H, Zhang J, Wu W, Dai L. Simultaneous quantification of serum monounsaturated and polyunsaturated phosphatidylcholines as potential biomarkers for diagnosing non-small cell lung cancer. Sci Rep. 2018;8:7137.

44. Klupczynska A, Plewa S, Kasprzyk M, Dyszkiewicz W, Kokot ZJ, Matysiak J. Serum lipidome screening in patients with stage I non-small cell lung cancer. Clin Exp Med. 2019;19:505-13.

45. Quehenberger $\mathrm{O}$, Armando AM, Brown AH, Milne SB, Myers DS, Merrill AH, Bandyopadhyay S, Jones KN, Kelly S, Shaner RL. Lipidomics reveals a remarkable diversity of lipids in human plasma. J Lipid Res. 2010;51:3299-305.

46. Cheng M, Bhujwalla ZM, Glunde K. Targeting phospholipid metabolism in cancer. Front Oncol. 2016;6:266.

47. Ogretmen B. Sphingolipid metabolism in cancer signalling and therapy. Nat Rev Cancer. 2018;18:33.

48. Huang W-C, Chen C-L, Lin Y-S, Lin C-F. Apoptotic sphingolipid ceramide in cancer therapy. J Lipid. 2011;2011:15.

49. Selzner M, Bielawska A, Morse MA, Rüdiger HA, Sindram D, Hannun YA, Clavien P-A. Induction of apoptotic cell death and prevention of tumor growth by ceramide analogues in metastatic human colon cancer. Cancer Res. 2001;61:1233-40.

50. Rylova S, Somova O, Dyatlovitskaya E. Comparative investigation of sphingoid bases and fatty acids in ceramides and sphingomyelins from human ovarian malignant tumors and normal ovary. Biochemistry (Mosc). 1998;63:1057-60.

51. Schiffmann S, Sandner J, Birod K, Wobst I, Angioni C, Ruckhäberle E, Kaufmann M, Ackermann H, Lötsch J, Schmidt H. Ceramide synthases and ceramide levels are increased in breast cancer tissue. Carcinogenesis. 2009;30:745-52.

52. Modrak DE, Lew W, Goldenberg DM, Blumenthal R. Sphingomyelin potentiates chemotherapy of human cancer xenografts. Biochem Biophys Res Commun. 2000;268:603-6.

53. Moro K, Kawaguchi T, Tsuchida J, Gabriel E, Qi Q, Yan L, Wakai T, Takabe K, Nagahashi M. Ceramide species are elevated in human breast cancer and are associated with less aggressiveness. Oncotarget. 2018;9:19874.

54. Kachler K, Bailer M, Heim L, Schumacher F, Reichel M, Holzinger CD, Trump S, Mittler S, Monti J, Trufa DI, et al. Enhanced acid sphingomyelinase activity drives immune evasion and tumor growth in non-small cell lung carcinoma. Cancer Res. 2017;77:5963-76. 
55. Kim HJ, Jang SH, Ryu J-S, Lee JE, Kim YC, Lee MK, Jang TW, Lee S-Y, Nakamura $\mathrm{H}$, Nishikata $\mathrm{N}$. The performance of a novel amino acid multivariate index for detecting lung cancer: a case control study in Korea. Lung Cancer. 2015;90:522-7.

56. Klupczynska A, Dereziński P, Dyszkiewicz W, Pawlak K, Kasprzyk M, Kokot ZJ. Evaluation of serum amino acid profiles' utility in non-small cell lung cancer detection in Polish population. Lung Cancer. 2016;100:71-6.

57. Shingyoji M, lizasa T, Higashiyama M, Imamura F, Saruki N, Imaizumi A, Yamamoto H, Daimon T, Tochikubo O, Mitsushima T. The significance and robustness of a plasma free amino acid (PFAA) profile-based multiplex function for detecting lung cancer. BMC Cancer. 2013;13:77.

58. Maeda J, Higashiyama M, Imaizumi A, Nakayama T, Yamamoto H, Daimon T, Yamakado M, Imamura F, Kodama K. Possibility of multivariate function composed of plasma amino acid profiles as a novel screening index for non-small cell lung cancer: a case control study. BMC Cancer. 2010;10:690.
59. Possemato R, Marks KM, Shaul YD, Pacold ME, Kim D, Birsoy K, Sethumadhavan S, Woo H-K, Jang HG, Jha AK. Functional genomics reveal that the serine synthesis pathway is essential in breast cancer. Nature. 2011;476:346.

60. Amelio I, Cutruzzolá F, Antonov A, Agostini M, Melino G. Serine and glycine metabolism in cancer. Trends Biochem Sci. 2014;39:191-8.

61. Abouleila Y, Onidani K, Ali A, Shoji H, Kawai T, Lim CT, Kumar V, Okaya S, Kato K, Hiyama E. Live single cell mass spectrometry reveals cancerspecific metabolic profiles of circulating tumor cells. Cancer Sci. 2019;110:697.

\section{Publisher's Note}

Springer Nature remains neutral with regard to jurisdictional claims in published maps and institutional affiliations.
Ready to submit your research? Choose BMC and benefit from:

- fast, convenient online submission

- thorough peer review by experienced researchers in your field

- rapid publication on acceptance

- support for research data, including large and complex data types

- gold Open Access which fosters wider collaboration and increased citations

- maximum visibility for your research: over $100 \mathrm{M}$ website views per year

At BMC, research is always in progress.

Learn more biomedcentral.com/submissions 